\title{
Mode Localization in Dynamics and Buckling of Linear Imperfect Continuous Structures
}

\author{
ANGELO LUONGO \\ Department of Structural Engineering, University of L'Aquila, 67040 Monteluco Roio, Italy
}

\begin{abstract}
Localization phenomena in one-dimensional imperfect continuous structures are analyzed, both in dynamics and buckling. By using simple models, fundamental concepts about localization are introduced and similarities between dynamics and buckling localization are highlighted. In particular, it is shown that strong localization of the normal modes is due to turning points in which purely imaginary characteristic exponents assume a non zero real part; in contrast, if turning points do not occur, only weak localization can exist. The possibility of a disturbance propagating along the structure is also discussed. A perturbation method is then illustrated, which generalizes the classical WKB method; this allows the differential problem to be transformed into a sequence of algebraic problems in which the spatial variable appears as a parameter. Applications of the method are worked out for beams and strings on elastic soil. All these structures are found to have nearly-defective system matrices, so their characteristic exponents are highly sensitive to imperfections.
\end{abstract}

Keywords: Localization, dynamics, buckling, perturbation methods, turning point, defective system.

\section{Introduction}

The mechanical behavior of high modal density structures can be considerably influenced by structural imperfections, even in the linear field. In particular, the normal modes of oscillations or the buckling modes, which in the absence of irregularities extend to the whole system, can localize in a restricted region of the structure. Thus, if an external disturbance is applied at a point, its effects cannot propagate very far but are confined to the neighborhood of the source.

Localization has been extensively analyzed for discrete systems. Pioneering studies were originally pursued in physics, by investigating disordered chains of atoms (see $[1,2]$ for a review) and many years later in mechanics, by analyzing chains of pendulums [3]. Extensive reviews on the subject are given in $[4,5]$.

In dynamics, attention has been mainly focused on discrete one-dimensional structures, such as open or closed chains of pendulums [3, 6-10] or bi-pendulums [11]. Periodic continuous systems such as chains of beams, multi-span beams and taut strings or cables with lumped masses have been analyzed through finite elements or Galerkin techniques [7, 8, 1215] or by exact transfer matrices [16-21]. Axially vibrating beams with or without distributed elastic constraints have been considered in $[22,23]$ by following a stochastic or deterministic approach, respectively. Nonlinear problems have been studied only recently $[24,25]$; in particular the existence of localized nonlinear normal modes has been proved in [26-28]. In [26] it has been observed that the origin of nonlinear mode localization in lumped-mass periodic systems is the dependence of the frequencies of substructures on their vibration amplitude; thus nonlinearities cause mistuning and localization takes place even in perfectly periodic systems. 
In contrast, localization in buckling has received little attention in the linear field [29] and considerable attention in the postcritical nonlinear analysis, where the phenomenon is explained to be a consequence of the interaction of a cluster of nearly-simultaneous modes. Many studies have been devoted to this subject; a review is presented in [30]. However, similarly to the dynamic problem, localization in buckling of perfect nonlinear structures has strict analogies with localization in buckling of imperfect linear structures, as it was observed in [31].

The localization phenomenon in linear continuous one-dimensional systems is considered here, both in dynamics and buckling. The problem is discussed with the following aims: (1) to improve the understanding of the mechanical phenomenon; (2) to emphasize that strong localization is closely connected with the occurrence of turning points [32], in absence of which only weak localization can occur; (3) to show that localization of vibrations and buckling patterns have common aspects; (4) to show that continuous structures undergoing strong localization are nearly-defective systems.

The paper is organized as follows. In Section 2 basic concepts on localization are introduced by referring to continuous systems, both in dynamics and buckling. In Section 3 a perturbation method for a general imperfect linear system with high modal density is illustrated. Three examples are worked out in Section 4: namely, the free vibrations of a string and of a beam resting on a Winkler soil, and the buckling pattern of a compressed beam on elastic soil. In Section 5, it is shown that, since the system matrices of these structures are nearly-defective (i.e. they have nearly-coincident characteristic exponents with associated nearly-parallel eigenvectors), their imperfection sensitivity is high. Therefore, small defects cause large variations in the characteristic exponents, thus possibly exalting the tendency towards localization.

\section{Basis Concepts}

Basic concepts about localization in continuous systems, both in dynamics and buckling, are illustrated and examples are given.

\subsection{LOCALIZATION OF VIBRATIONS}

Let us consider a continuous one-dimensional structure with spatially constant mechanical properties (perfect system) vibrating at an undamped natural frequency. Let us assume first that under suitable boundary conditions the (spatial) modal shape is sinusoidal, i.e. a couple of characteristic exponents of the differential equation of motion is purely imaginary. If some spatially-dependent imperfection is introduced in the system, such characteristic exponents are perturbed from their nominal value, and assume new values that depend on the site. If they have a non zero real part throughout most of the structure, and if the structure is sufficiently long, the modal shape localizes. The points at which the characteristic exponents change from imaginary to real or complex conjugate are called turning points. If more general boundary conditions are prescribed, even the perfect system has real or complex characteristic exponents contributing to the eigenfunctions. However, the exponents are independent of the site and significantly affect the solution only in narrow boundary layers, except perhaps for the first few modes. When sufficiently large imperfections are introduced, the real parts exponents depend on the site and, through the mechanism previously illustrated, can enforce localization to occur 
somewhere in the dominion. In order to separate the effects of boundary conditions from that of imperfections, reference will be made to systems having purely sinusoidal eigenfunctions.

The occurrence of turning points represents the simplest mechanism which triggers localization. However, it is not the only way for a normal mode to localize. As an example, the axially vibrating beam studied in [22] can undergo localization albeit its characteristic exponents are always purely imaginary, independently from the mass and/or stiffness imperfection shape. For such kind of structures, localization is due to the fact the amplitude of the oscillating modal shape decays exponentially in the space, as a result of the changes of the mechanical properties along the structure. Thus, if the imperfections are stepwise variable, the normal modes are made of pieces of sinusoids with different amplitudes and phases. If the amplitudes tend to zero far away from a certain section of the structure, then the mode is localized. This circumstance always occurs in a sufficiently long structure and it is strictly connected with the random nature of the imperfections (see, e.g., [2], where the Furstemberg theorem concerning the limit of noncommuting random variables is used to explain the phenomenon).

The two mechanisms of localization illustrated are quite different and should not be confused. Moreover, while the first one (when it can occur) leads very directly to localization, the second one usually calls for longer structures and/or larger random disorder. For this reason the two phenomena could be referred to as strong localization and weak localization, thus more sharply defining a difference already discussed by Pierre [9], although from a different point of view. According to that paper, weak localization has little interest in engineering structures; therefore, attention is mainly paid here to strong localization. Hence, the word 'localization' will be used as shortening of 'strong localization'.

To illustrate the main aspects of the phenomenon, reference is made to a specific problem, already analyzed in [23]. The main findings are resumed here and new results are illustrated.

Consider a string weakly taut by a small force $T$, having mass per unit length $m_{0}$, resting on a Winkler soil of nominally constant stiffness $k_{f}=k_{0}$. The perfect system has sinusoidal modes with close frequencies. The problem is to determine the modes and the frequencies of the imperfect system when small defects affect the stiffness of the springs, i.e. when $k_{f}=$ $k_{f}(x)$ depends on the abscissa $x$ [23]. The normal modes $w(x)$ of a fixed-fixed string satisfy the following homogeneous boundary value problem

$$
\begin{aligned}
& \beta^{2} w^{\prime \prime}+(\lambda-\chi(x)) w=0, \quad x \in[-1 / 2,1 / 2], \\
& w(-1 / 2)=0, \quad w(1 / 2)=0,
\end{aligned}
$$

in which $\beta=\left(T / k_{0} l^{2}\right)^{1 / 2} \ll 1$ is the coupling parameter, responsible for the high modal density of the perfect system, $\lambda=m_{0} \omega^{2} / k_{0}$ is the nondimensional frequency and $\chi(x)=$ $k_{f}(x) / k_{0}$ is the nondimensional stiffness of the imperfect springs.

Equation (1a) can easily be integrated if a single defect consisting in a rectangular stiffness well centered at the midspan $x=0$ is present. In this case $\chi(x)=1-\varepsilon$ for $0 \leq|x|<\Delta$ and $\chi(x)=1$ for $\Delta<|x| \leq 1 / 2$, where $\varepsilon$ and $2 \Delta$ are the depth and the width of the well, respectively. Correspondingly the solution $w(x)$ has two branches, $w_{i}(x)=\exp \left(\theta_{i} x\right)$ and $w_{e}(x)=\exp \left(\theta_{e} x\right)$, internal and external to the well, respectively. Two cases must be distinguished:

(a) When $\lambda>1$, both $w_{i}(x)$ and $w_{e}(x)$ are sinusoidal functions, so the associated normal mode is extended, as for the perfect structure. The $a_{e} / a_{i}$ ratio between the amplitudes of the two branches is found to be always larger than 1, so that no weak localization occurs. However, 

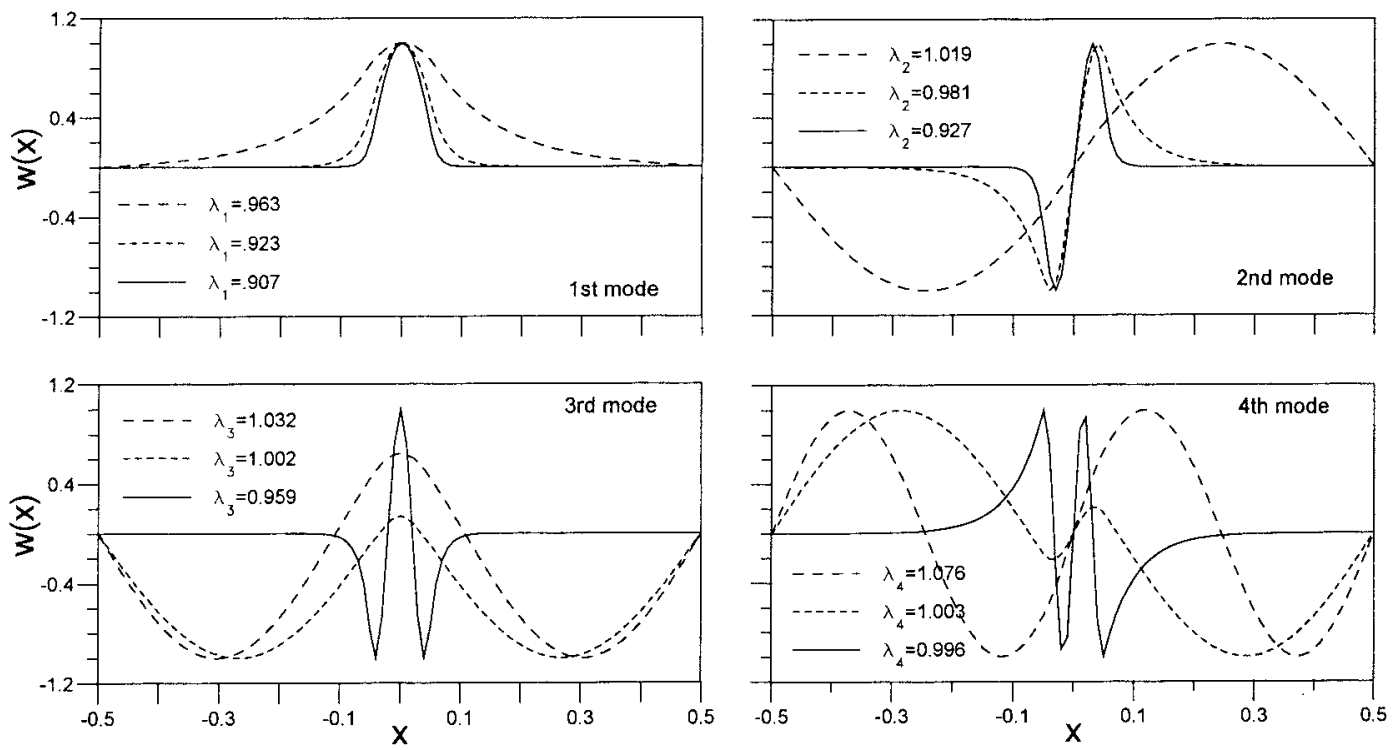

(a)

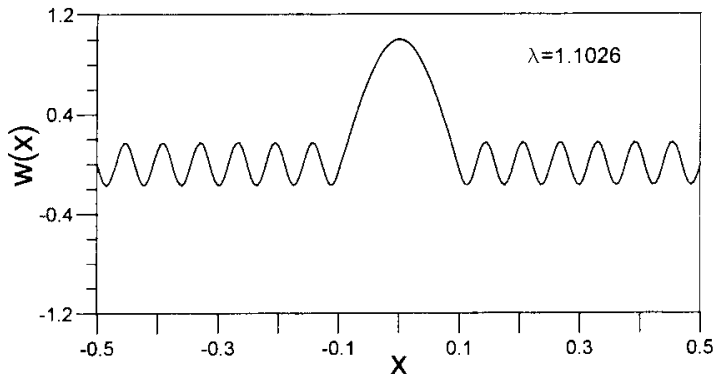

(b)

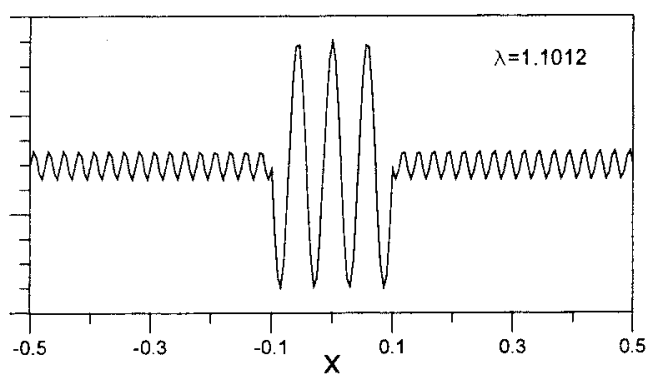

(c)

Figure 1. Normal modes for the string on elastic soil with rectangular stiffness well (a) or peak (b) centered at midspan; (a) $\varepsilon=0.1, \Delta=0.05,--\beta=0.02236, \cdots \beta=0.00707,-\beta=0.00316$; (b) $\varepsilon=-0.1, \Delta=0.1$, $\beta=0.00316$; (c) $\varepsilon=-0.1, \Delta=0.1, \beta=3.16 \times 10^{-4}$.

if $\varepsilon<0$ (i.e. if a peak of soil stiffness is considered instead of a well), $a_{e} / a_{i}<1$ and weak localization can take place.

(b) When $1-\varepsilon<\lambda<1, w_{i}(x)$ is sinusoidal while $w_{e}(x)$ is exponential, so that the mode is localized near the defect; in this case the character of the solution changes at the point $x=\Delta$, which is a turning point. As can easily be checked from Equation (1a), the following relation between the characteristic exponents $\theta_{i}$ (purely imaginary) and $\theta_{e}$ (real) holds: $\left|\theta_{i}\right|^{2}+\theta_{e}^{2}=\mu$, where $\mu=\varepsilon / \beta^{2}$ is the imperfection/coupling ratio. The relation shows that $\left|\theta_{i}\right|$ and $\theta_{e}$ range between zero and $\sqrt{\mu}$. Thus, since the first modes are associated with smaller values of $\left|\theta_{i}\right|$, they also have greater values of $\theta_{e}$, therefore lower modes are more strongly localized than higher modes. Because $\left|\theta_{i}\right|$ is limited, the number of localized modes is limited as well, and increases with $\mu$. If $\mu$ is very small no localized modes can occur. Therefore, in a finite length system, a threshold defect must exist in order to localize a given number of eigenfunctions; on the other hand, if the length is sufficiently large, any small defect can localize the first modes. 


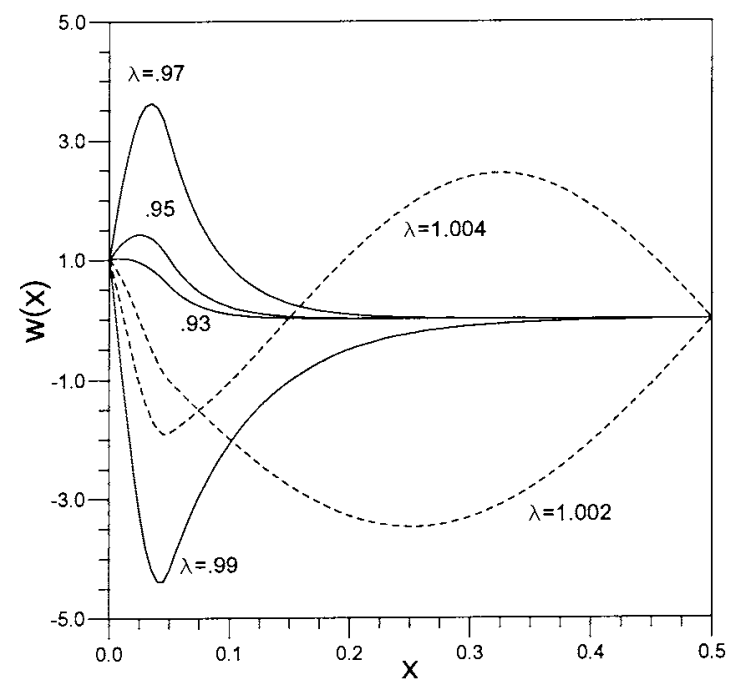

Figure 2. Time-harmonic response of the second string in Figure 1a when the midspan is driven with frequency $\lambda ; \Delta=0.05, \varepsilon=0.1, \beta=0.00707$.

Some normal modes for different values of the parameters are plotted in Figure 1. Figure 1a shows the effects of the occurrence of turning points. It is seen that, for a fixed imperfection, the number of localized modes increases when $\beta$ decreases. Thus, while the first mode is localized for all $\beta$ 's considered, the fourth mode localizes only for the smallest $\beta$. In Figures $1 \mathrm{~b}$ and 1c some weakly localized mode produced by a peak of stiffness are shown. They occasionally occur in sequences of nonlocalized modes. To observe localization of more modes it would be necessary to introduce random diffused imperfections.

Examine now the possibility of a disturbance propagating along the string. To this end, assume that the midspan $x=0$ is harmonically driven by a device with a known frequency $\lambda$ and unitary amplitude. By accounting for the antisymmetry of the problem, the amplitude $w(x)$ of the time-harmonic response is determined by integrating Equation (1a) in the interval $[0,1 / 2]$ with the boundary conditions $w(0)=1, w(1 / 2)=0$. The response is plotted in Figure 2 for various $\lambda$; the string is the second of those considered in Figure 1a. It is apparent that, when $\lambda<1$, due to the presence of a turning point at $x=\Delta$, the response is localized around the driven point; when $\lambda>1$, since no turning points occur, the response is extended to the whole string. It should be noted that resonance occurs when $\lambda$ is equal to $0.981,1.003, \ldots$ i.e. when the frequency of the excitation is equal to a natural frequency of an antisymmetric mode of the double length string (see Figure 1a). Thus, when $\lambda$ is equal to 0.97 or 0.99 , the response is essentially in the first mode, while when $\lambda$ is equal to 1.002 or 1.004 the second mode prevails. However, since the first mode is localized, the first disturbances cannot propagate; in contrast, since the second mode is extended, the last disturbances propagate. 


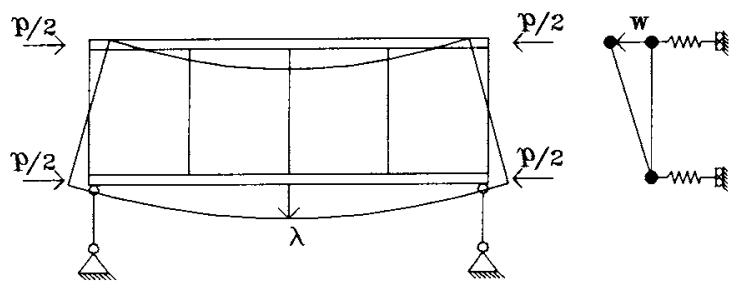

(a)

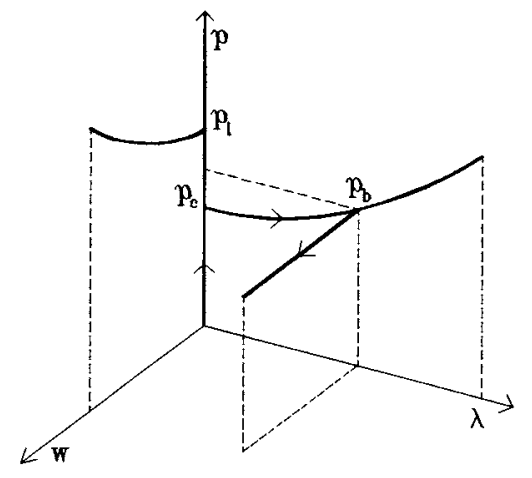

(b)

Figure 3. Truss structure elastically restrained; (a) model, (b) equilibrium paths.

\subsection{Localization of BuCKLing Patterns}

It is well known from the theory of elastic stability that simple compressed structures such as long beams on elastic soil, long plates, thin-walled beams or spheres under hydrostatic pressure exhibit a large number of nearly coincident buckling modes, i.e. they have dense spectra. This is due to the fact that a small parameter $\beta$-typically an aspect ratio - appears in the eigenvalue equation. As a consequence, if imperfections were taken into account in the analysis, localization should occur. To prove that, a simple model similar to the previous one could be considered, consisting in a uniformly compressed beam on imperfect elastic soil. However, in spite of the formal analogy with the dynamical problem, localization in buckling does not seem to have been studied in depth. In fact, the imperfections that are usually accounted for in elastic stability are typically small curvatures or eccentricities of the forces (the so-called 'initial imperfections'), instead of small spatial variations of the mechanical characteristics, as thicknesses or material properties. Since the former imperfections add only known terms to the boundary value problem relevant to the perfect system, they leave its spectral properties unaltered and localization cannot occur. In contrast, the latter imperfections leave the boundary value problem homogeneous and therefore modify the eigensolutions, eventually allowing localization.

On the other hand, a large amount of literature exists on the subject of nonlinear interaction among nearly simultaneous buckling modes. In particular, it has been shown that buckling patterns in the postcritical range can localize in one or more regions, in contrast with the periodic character of the critical modes [33-37]. A simple model studied by the author in [31] clarifies the fact the mechanism which produces localization in nonlinear perfect systems is the same as that which produces localization in linear imperfect systems. In other words, nonlinearities have the same effects as imperfections.

The example given in [31] concerns a simply supported planar truss-beam, having continuous top and bottom longerons which are restrained against out-of-plane displacements by distributed elastic springs (Figure 3a). When the structure is compressed, it can buckle in an overall (Eulerian) mode or in several out-of-plane local modes whose wavelength is small compared with the span. The system behaves as a compressed thin-walled beam that in fact buckles in an overall (Eulerian or flexural-torsional) mode or in a local mode in which the component plates are bent in many longitudinal waves. It is assumed here that the lower 
critical load multiplier $p_{c}$ is associated with the Eulerian mode and the higher critical load $p_{l}$ with the local mode (Figure $3 \mathrm{~b}$ ). When the load $p$ is increased, the structure first buckles in its plane at $p=p_{c}$ (primary bifurcation), then it buckles out-of-plane at $p=p_{b}$ (secondary bifurcation); as a result of the interaction it ensues that $p_{b}<p_{l}$. The goal of the analysis is to evaluate the secondary bifurcation load $p_{b}$ along the non trivial bifurcated equilibrium path, together with the associated modal shape. It is assumed that $p_{l}$ is slightly higher than $p_{c}$, so the problem can be linearized in the nondimensional amplitude $\lambda$ of the overall mode, which is taken as being the control parameter.

The problem is governed by the classical equilibrium equation of the compressed beam resting on Winkler soil. Along the trivial fundamental path the axial force in the two longerons is constant; along the bifurcated path, due to the bending associated with the primary bifurcation, an additional spatially variable traction or compression exists in the two beams. For the most compressed beam, the boundary value problem reads

$$
\begin{aligned}
& \beta^{4} w^{\prime \prime \prime \prime}+2 \beta^{2}\left[(p+\lambda f(x)) w^{\prime} / p_{l}\right]^{\prime}+w=0, \quad x \in[0, \pi], \\
& w(0)=w^{\prime \prime}(0)=0, \quad w(\pi)=w^{\prime \prime}(\pi)=0 .
\end{aligned}
$$

In Equation (2a) $\beta=\pi\left(E I / k_{0} l^{4}\right)^{1 / 4}$, where $E I$ is the out-of-plane flexural stiffness of the longerons, $l$ is their length and $k_{0}$ is the stiffness of the springs; since $\beta$ equals the ratio between the wavelengths of the local and overall modes it is, by hypothesis, a small parameter. In addition, in Equation (2a), $f(x)=\sin x$ accounts for the additional variable compression whose maximum value is assumed to be (approximately) proportional to the overall deflection $\lambda$.

Along the trivial path $\lambda \equiv 0$, and $p$ represents the unknown eigenvalue. The problem (2) admits the solution $p=p_{l}$ and $w=\sin (n x)$, with $n \cong \beta^{-1}$. Other eigenvalues slightly higher than $p_{l}$ correspond to sinusoidal modes with wavenumbers $n \pm k$, with $k \ll n$, so that the system has high modal density.

Along the bifurcated path $\lambda \neq 0, p$ depends on $\lambda$; since the Eulerian bifurcation is symmetric and $\lambda$ is a small quantity, $p=p_{c}+O\left(\lambda^{2}\right)$. Equations (2) are therefore an eigenvalue problem in the displacement $\lambda$. By posing $p_{c}=1, p_{l}=1+\varepsilon$, where $\varepsilon>0$ is a small parameter, and retaining only first-order quantities, Equations (2) become

$$
\begin{aligned}
& \beta^{4} w^{\prime \prime \prime \prime}+2 \beta^{2}\left[(1-\varepsilon) w^{\prime \prime}+\lambda\left(f(x) w^{\prime}\right)^{\prime}\right]+w=0, \quad x \in[0, \pi], \\
& w(0)=w^{\prime \prime}(0)=0, \quad w(\pi)=w^{\prime \prime}(\pi)=0 .
\end{aligned}
$$

The parameter $\varepsilon$, measuring the mistuning between the two critical loads $p_{c}$ and $p_{l}$, represents a 'defect' in the broad sense. In fact, when $\varepsilon=0$, i.e. when the two critical loads are tuned, Equation (3) admits the solution $\lambda=0$ and $w=\sin (n x)$ : there is therefore no reduction (the so-called erosion [30]) in the local critical load. In contrast, if $\varepsilon \neq 0, \lambda$ can be shown to be of order $\varepsilon$ [31], so that $p_{b}=1+O\left(\varepsilon^{2}\right)<p_{l}$, and erosion takes place.

It should be noted that, unlike the example dealt with before and in the remainder of the paper, the parameter $\varepsilon$ does not affect the part of the differential operator depending on $x$. Thus, when $\varepsilon=0$, Equation (3a) does not reduce to a constant coefficient equation, if $\lambda=0$ is not posed as well. However, by scaling the eigenvalue at the order $\varepsilon$, i.e. by posing $\lambda=\varepsilon \sigma$, with $\sigma=O(1)$, this condition is satisfied. In this form Equation (3) appears as a perturbation of order $\varepsilon$ of a singular operator with constant coefficients. The perturbation assumes the meaning of an imperfection, due to the nonlinearities, of the geometrical stiffness of the beam. 
Since the perfect system has high modal density, it can be predicted that this defect produces localization, as do defects in the true sense of the word.

To evaluate the critical load and the bifurcation mode it is necessary to integrate Equation (3a); however exact integration is not straightforward, as it was in the previous example. An asymptotic solution has been found in [31] by using the multiple scale perturbation method to obtain an amplitude modulating equation. This equation turned out to be exactly of the same form of Equation (1a), with a sinusoidal coefficient. By applying the WKB method [32], amplitude modulating solutions qualitatively similar to the ones in Figure 1a were obtained, still exhibiting turning points, although no more in a priori known positions. The localized buckling patterns therefore appear like those in Figure 10 below.

To summarize, in this system nonlinearities play the same role as imperfections in linear problems and, together with the high modal density of the structure, cause localization of the buckling patterns. As has been explained in Section 1, a similar result was found in [26] for a discrete dynamical system.

\section{A General Perturbation Method for Continuous Systems}

The examples analyzed before have shown that, in order to evaluate the normal modes of an imperfect continuous system and to check the possible occurrence of localization, it is necessary to integrate a differential equation whose coefficients are weakly dependent on the abscissa $x$. Since exact integration is often impossible, it would be desirable to take advantage of the presence in the equation of a small parameter to obtain asymptotic solutions. However, it is known that series expansions in terms of the imperfection parameter $\varepsilon$ are not suited to the scope $[6,7]$. In fact, due to the presence of small divisors, asymptotic expansions obtained by perturbing a unique eigenfunction of the perfect system are not uniformly valid. On the other hand, perturbations of a cluster of eigenfunctions of the perfect system lead to a new eigenvalue problem in the unknown amplitudes [38], thus practically nullifying the convenience of using a perturbation method. A more suitable method has been used by Pierre and co-workers $[6,8,14]$ in which the structural parameter $\beta$, instead of the imperfection parameter $\varepsilon$, has been adopted as the perturbation parameter. This method can be successfully applied for discrete structures but not for a continuous structure, as will be explained ahead.

A general perturbation method for the analysis of one-dimensional linear continuous structures with high modal density, affected by small imperfections, is illustrated here. The method applies to boundary value problems that can be put in the form

$$
\begin{aligned}
\beta \frac{\mathrm{d} w}{\mathrm{~d} x} & =L(x, \varepsilon, \lambda) w, \quad x \in[0,1], \\
B_{0} w(0) & =w_{0}, \quad B_{1} w(1)=w_{1},
\end{aligned}
$$

where $\beta$ and $\varepsilon$ are small parameters, $\lambda=O(1)$ is a control parameter, $w$ is the $N$-state-vector containing generalized displacements and stresses, $L$ is the $N \times N$ system matrix, $B_{0}$ and $B_{1}$ are Boolean $(N / 2) \times N$ matrices, and $w_{0}$ and $w_{1}$ are prescribed values of the state variables at the ends of the structure.

The parameter $\lambda$ has the meaning of a time-frequency in dynamics and a load multiplier (or a nondimensional displacement) in buckling problems. It is the unknown eigenvalue of the differential problem when the boundary conditions are homogeneous $\left(w_{0}=w_{1}=0\right)$ or assumes known values when non zero displacements and/or stresses are prescribed as external 
disturbances at the ends of the structure. The parameter $\beta$ is a structural parameter, depending on the mechanical characteristics of the perfect structure, and is responsible for the high modal density. The parameter $\varepsilon$ is the imperfection parameter, measuring the amplitude of the defects. When $\varepsilon=0$ the structure is perfect and, by hypothesis, has mechanical properties independent of the abscissa $x$, so that the matrix $L^{*}=L(x, 0, \lambda)$ has constant coefficients. By expanding $L$ around $\varepsilon=0$ it follows

$$
L(x, \varepsilon, \lambda)=L^{*}(\lambda)+\varepsilon L_{\varepsilon}(x, \lambda)+O\left(\varepsilon^{2}\right) .
$$

From now on the dependence on $\lambda$ of all the variables will be taken as understood.

Consider first the perfect system $(\varepsilon=0)$. Equation (4a) admits solutions of the type

$$
w(x, \beta)=u^{*} \exp \left(\frac{\theta^{*}}{\beta} x\right),
$$

where $u^{*}$ and $\theta^{*}$ are generally complex solutions of the $N \times N$ algebraic eigenvalue problem

$$
\left(L^{*}-\theta^{*}\right) u^{*}=0 .
$$

The eigenvalues $\theta^{*}$ are the characteristic exponents of the differential equation (4a). If $L^{*}$ admits a complete set of eigenvectors $u^{*}$, the general solution to Equation (4a) can be built as a linear combination of the solutions (6). If homogeneous boundary conditions are enforced, a transcendental equation in $\lambda^{*}$ is obtained. By hypothesis let

$$
\lambda_{k}^{*}=1+O\left(\beta^{h}\right), \quad h>1
$$

be the eigenvalues of interest for $k$ ranging over some interval. When $\beta$ is small the system has high modal density, increasing with the exponent $h . \beta^{h}$ is a measure of the spread in the eigenvalues of the perfect system. From Equation (6), if the associated characteristic exponents $\theta_{k}^{*}$ have modulus $\left|\theta_{k}^{*}\right|=O(1)$, the eigenfunctions are fast varying; if $\left|\theta_{k}^{*}\right|=O(\beta)$, they are slowly varying.

Consider now the imperfect system and look for an asymptotic solution. Since two small parameters $\beta$ and $\varepsilon$ are present in the equation, attention will be focused on the case in which the imperfections have amplitude greater than, or equal to, the bandwidth of the eigenvalues $\lambda_{k}^{*}$, i.e., from Equation (8), when $\varepsilon \geq O\left(\beta^{h}\right)$.

Series expansions could be performed in terms of either $\beta$ or $\varepsilon$, by fixing one parameter and expanding with respect to the other. However, if $\beta$ is fixed and $\varepsilon \rightarrow 0$, a large number of nearly-simultaneous modes $u_{k}^{*}$ has to be considered to express the generating solution, as has already been discussed. In contrast, if $\varepsilon$ is fixed and $\beta \rightarrow 0$, the nearly-degenerate condition of the generating system is destroyed and a perturbation solution can be pursued in a simpler way. However, since $\beta$ affects the highest derivative in Equation (4), a singular perturbation problem arises and standard method cannot be applied.

A similar question occurs when discrete periodic structures are considered, like chains of weakly coupled one d.o.f. subsystems. Pierre and co-workers $[6,8,14]$ have shown that asymptotic expansions in terms of the coupling parameter $\beta$ can be successfully employed to determine the eigenfunctions of the perturbed system and to explain the phenomenon of localization. Their generating equation has been obtained by posing $\beta=0$ in the governing equations, i.e. by considering the uncoupled subsystems as a generating mechanical system. The same operation cannot be performed when continuous systems are dealt with, since kinematic compatibility would be violated. The method presented here enables this difficulty to be overcome. 
Since Equation (4a) has variable coefficients, a solution is still sought in the form (6), in which, however, $u$ and $\theta$ are assumed to depend on $x$, i.e.

$$
w(x, \beta, \varepsilon)=u(x, \beta, \varepsilon) \exp \left\{\frac{1}{\beta} \int \theta(x, \beta, \varepsilon) \mathrm{d} x\right\} .
$$

Moreover, $u$ and $\theta$ are expanded in series of the perturbation parameter $\beta$ around $\beta=0$

$$
\begin{aligned}
& u(x, \beta, \varepsilon)=\sum_{j=0}^{\infty} \beta^{j} u_{j}(x, \varepsilon), \\
& \theta(x, \beta, \varepsilon)=\sum_{j=0}^{\infty} \beta^{j} \theta_{j}(x, \varepsilon) .
\end{aligned}
$$

By substituting Equations (9) and (10) in Equation (4a) and zeroing the terms with the same powers of $\beta$, the following perturbation equations are drawn

$$
\begin{aligned}
& \left(L-\theta_{0}\right) u_{0}=0, \\
& \left(L-\theta_{0}\right) u_{1}=-u_{0}^{\prime}-\theta_{1} u_{0}, \\
& \left(L-\theta_{0}\right) u_{2}=-u_{1}^{\prime}-\theta_{1} u_{1}-\theta_{2} u_{0}, \\
& \ldots,
\end{aligned}
$$

where all quantities depend on $x$ and $\varepsilon$, in addition to $\lambda$, and a prime denotes differentiation with respect to $x$.

It should be noted that the original differential problem has been transformed in a sequence of algebraic problems in which the abscissa $x$ appears as a parameter. Equation (11a) is the generating equation; it is still an eigenvalue problem, like Equation (7), however it furnishes spatially variable characteristic exponents $\theta_{0}$.

It is supposed that the system matrix $L$ has $N$ distinct eigenvalues; let $\theta_{0}$ be one of these eigenvalues, with associated right eigenvector $u_{0}$ and left eigenvector $v_{0}$ (i.e. $L^{H} v_{0}=\bar{\theta} v_{0}$, where the overbar denotes the complex conjugate and the superscript $H$ the transpose conjugate). By substituting $u_{0}$ and $\theta_{0}$ in Equation (11b) a nonhomogeneous linear equation with singular coefficient matrix is obtained. The solvability condition requires the right-hand member to be orthogonal to the left eigenvector $v_{0}$ from which the unknown $\theta_{1}$ is drawn

$$
\theta_{1}=-v_{0}^{H} u_{0}^{\prime} / v_{0}^{H} u_{0} .
$$

Equation (11b) can then be solved to within an arbitrary constant and the procedure be continued to higher orders.

After having determined $N$ solutions in this way, the general solution is obtained as a linear combination. By enforcing homogeneous boundary conditions the eigenvalues $\lambda_{k}$ and the eigenfunctions $w_{k}(x)(k=1,2, \ldots)$ are evaluated. If nonhomogeneous boundary conditions are instead prescribed, for any given $\lambda$ different from the eigenvalues $\lambda_{k}$ the response $w(x)$ is calculated. However, in both cases, the method can furnish solutions that are not uniformly valid in the whole dominion, due to the occurrence of turning points [32]; the problem will be illustrated by reference to applications. 


\section{Illustrative Examples}

Vibrations and buckling patterns of three imperfect one-dimensional structures resting on Winkler soil are analyzed by using the perturbation method developed in Section 3. The tendency towards localization is studied.

\subsection{Free and Forced Vibrations of a Weakly taut long String on Elastic SOIL}

The string on elastic soil is considered again, with imperfections affecting the foundation stiffness only. The ends of the string are fixed (free vibrations) or are subjected to timeharmonic transversal displacements (forced vibrations). Harmonic motions of frequency $\omega$ are governed by the following boundary value problem

$$
\begin{aligned}
& T w^{\prime \prime}-k_{f}(x) w+m_{0} \omega^{2} w=0, \\
& w(0)=w_{A}, \quad w(l)=w_{B},
\end{aligned}
$$

where

$$
k_{f}(x)=k_{0}(1+\varepsilon f(x)),
$$

in which $f(x)$ accounts for the shape of the imperfections, normalized as $\max (|f(x)|)=1$.

By introducing the following nondimensional quantities

$$
\tilde{w}=\frac{w}{l}, \quad \tilde{x}=\frac{\pi x}{l}, \quad \beta=\frac{\pi}{l} \sqrt{\frac{T}{k_{0}}}, \quad \lambda=\frac{m_{0} \omega^{2}}{k_{0}}
$$

and omitting for simplicity the tilde, Equation (13a) can be recast in the normal form (4a)

$$
\beta\left\{\begin{array}{l}
w_{1}^{\prime} \\
w_{2}^{\prime}
\end{array}\right\}=\left(\left(\begin{array}{cc}
0 & 1 \\
1-\lambda & 0
\end{array}\right)+\varepsilon\left(\begin{array}{cc}
0 & 0 \\
f(x) & 0
\end{array}\right)\right)\left\{\begin{array}{l}
w_{1} \\
w_{2}
\end{array}\right\}
$$

in which $w_{1}$ is the deflection and $w_{2}$ the transversal component of the internal force in the deformed configuration. In addition $\beta \ll 1$ if $T \ll k_{0} l^{2}$, i.e. if the stress is small and/or the length is great.

The perfect system $(\varepsilon=0)$ is first considered. The eigenvalues of the matrix $L^{*}$ are $\theta^{*}=$ $\pm i \sqrt{\lambda-1}$. By enforcing the boundary conditions $w(0)=w(\pi)=0, \lambda_{k}^{*}=1+k^{2} \beta^{2}$ is found and, consequently, $\theta_{k}^{*}= \pm i k \beta(k=1,2, \ldots)$ (Figure 4a). Thus the system has high modal density with exponent $h=2$ (see Equation (8)) and sinusoidal eigenfunctions with characteristic exponents $\left|\theta_{k}^{*}\right|=O(\beta)$, i.e. slowly varying solutions.

The imperfect system is then studied. Imperfections are assumed to have amplitudes $\varepsilon \geq$ $O\left(\beta^{2}\right)$. The generating Equation (11a) admits the following eigenvalues

$$
\theta_{0}(x)= \pm \sqrt{\varepsilon(f(x)-\sigma)}
$$

where

$$
\lambda=1+\varepsilon \sigma, \quad \sigma=O(1)
$$

has been posed. The two characteristic exponents $\theta_{0}$ are real for any $x$ for which $f(x)>\sigma$ (i.e. for $k_{f}(x) / k_{0}>\lambda$ ) and imaginary for any $x$ for which $f(x)<\sigma$ (i.e. for $k_{f}(x) / k_{0}<\lambda$ ). 


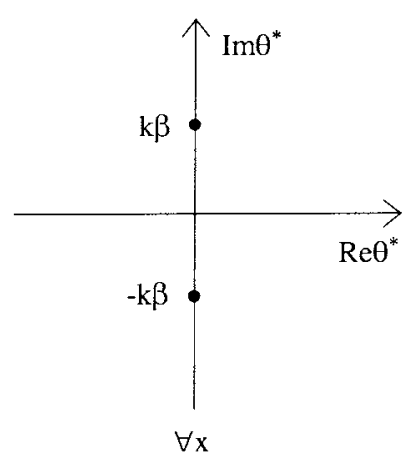

(a)

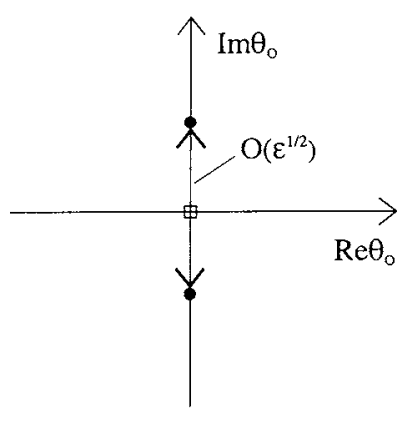

$\mathrm{f}(\mathrm{x})<\sigma$

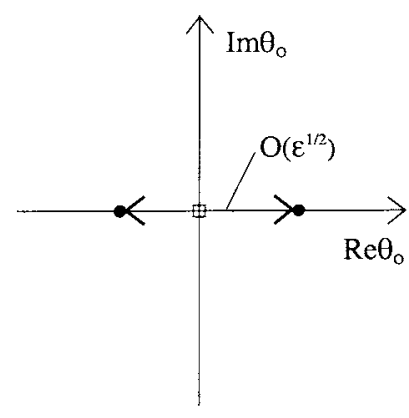

$f(x)>\sigma$

(b)

Figure 4. Characteristic exponents for the string on elastic soil; (a) perfect system $(\varepsilon=0)$, (b) imperfect system $(\varepsilon \neq 0) ; \square$ exponents at turning points.

The paths of the eigenvalues on the complex plane for $x$ varying are shown in Figure 4b. When $f(x)=\sigma$ the eigenvalues coalesce at the origin and $x$ is a turning point. It should be noted that $0 \leq\left|\theta_{0}(x)\right| \leq O\left(\varepsilon^{1 / 2}\right)$, so, for mode numbers $k=O(1)$, the imperfections produce corrections of the characteristic exponents $\theta^{*}$ of the same order as, or larger than, $\theta^{*}$, since $\varepsilon \geq O\left(\beta^{2}\right)$. The corresponding eigenfunctions are therefore strongly imperfection sensitive.

Right and left eigenvectors of $L$ are

$$
u_{0}=\left\{\begin{array}{ll}
1 & \theta_{0}
\end{array}\right\}^{T}, \quad v_{0}=\left\{\begin{array}{ll}
\bar{\theta}_{0} & 1
\end{array}\right\}^{T}
$$

and Equation (12) yields

$$
\theta_{1}(x)=-\frac{1}{2} \frac{\theta_{0}^{\prime}(x)}{\theta_{0}(x)}=\frac{\mathrm{d}}{\mathrm{d} x} \ln \theta_{0}^{-1 / 2}(x) .
$$

By using Equations (17), (19a) and (20) in Equations (9) and (10) the general solution

$$
\begin{aligned}
& w(x)=\frac{1}{\sqrt[4]{\sigma-f(x)}}\left\{c_{1} \sin \psi(x)+c_{2} \cos \psi(x)\right\} \quad \text { if } f(x)<\sigma, \\
& w(x)=\frac{1}{\sqrt[4]{f(x)-\sigma}}\left\{c_{3} \exp \psi(x)+c_{4} \exp (-\psi(x))\right\} \quad \text { if } f(x)>\sigma
\end{aligned}
$$

is obtained, with

$$
\psi(x)=\int \sqrt{\mu|f(x)-\sigma|} \mathrm{d} x .
$$

In Equations (21) $c_{i}$ are arbitrary constants and $\mu=\varepsilon / \beta^{2} \geq O(1)$ is the imperfection/coupling ratio. From the boundary conditions a linear system in the constant $c_{i}$ with coefficient dependent on $\sigma$ is obtained. The truncated asymptotic solution (21) is known as Liouville-Green or WKB approximation [32].

It should be noted that the solution (21) is singular at the turning points. Since their position depends on $\sigma$, they are unknown in a free vibration problem, but are a priori known in a forced vibration problem. To eliminate the singularity, polynomial (inner) solutions must be found around the singularities and successively matched with the (outer) solutions (21) that are valid 
far from the singular points. The matching procedure allows the arbitrary constant to be reduced to two and renders the boundary value problem well posed. The technique is illustrated in [32] and an application relative to a problem with a unique turning point has been developed by the author in [23]. However, for general imperfections, several turning points occur and the solution has oscillatory branches (Equation (21a)) and exponential branches (Equation (21b)) that alternate. Since the exponential decaying is proportional to $\mu^{1 / 2}$ (Equation (22)), if $\mu$ is sufficiently large the eigenfunctions localize around the stiffness gaps. If $\mu$ is not large enough, no turning points occur and the modal shapes remain oscillating and extended. This is always true for higher modes, for which $\sigma>\max (f(x)) \leq 1$, even when the lower modes are localized.

When many turning points are present, the matching analytic procedure becomes impracticable and it is necessary to resort to numerical methods. However, even in these cases, the perturbation method allows the acquisition of qualitative information. In particular it permits determination of the conditions under which disturbances prescribed at one end of the string cannot propagate to the other end, remaining localized near the source.

In Figure 5 the lowest modes of the string for different values of the parameters are shown. They have been obtained numerically by direct application of the Galerkin procedure. A single halfwave sinusoidal imperfection is considered first (Figures $5 \mathrm{a}$ and $5 \mathrm{~b}$ ), for which $k_{f}(x) / k_{0}$ ranges in $[0.9,1]$. For the lower value of $\mu$ (Figure 5a) the first two modes (associated with eigenvalues $\lambda<1$ ) exhibit two turning points while the third mode (with $\lambda>1$ ) is oscillatory. For the higher value of $\mu$ (Figure $5 \mathrm{~b}$ ) all the three modes have exponential branches near the ends. It is observed that the modes tend to localize around $x=\pi / 2$, i.e. around the point in which the stiffness of the soil is minimum. Localization would be magnified if $\mu$ were further increased. However, by choosing another shape of the imperfections, localization just manifests itself for the same values of $\mu$. In Figures $5 \mathrm{c}$ and $5 \mathrm{~d}$ the same string but on an elastic soil with two gaps of stiffness, each located near one end (cosinusoidal imperfection), is considered. The stiffness $k_{f}(x) / k_{0}$ now ranges in [0.9, 1.1]. For the lower $\mu$ (Figure 5c), the first two modes (with $\lambda<1.1$ ) are localized around the two gaps, that vibrate in-phase or outof-phase, while the higher modes (with $\lambda>1.1$ ) are extended. For the higher $\mu$ (Figure $5 \mathrm{~d}$ ), the first eight modes have turning points, the first four of which, more strongly localized, are shown. It should be noted in this last case, that symmetric and antisymmetric localized modes have nearly-coincident frequencies, since the coupling between the two gaps is very small. In conclusion, the numerical results show that localization strongly depends on the shape $f(x)$ of the imperfections. It is more pronounced for the second imperfection (Figures $5 \mathrm{c}$ and $5 \mathrm{~d}$ ) compared with the first imperfection (Figures 5a and 5b) because, due to the narrower gaps, the turning points are closer each other and, due to the greater difference $f(x)-\sigma$ out of the gaps, the exponential decaying is higher.

In Figure 6, the response of the string to an harmonic transversal excitation of frequency $\lambda$ of the left support is shown; the parameters are the same as in Figures $5 \mathrm{c}$ and $5 \mathrm{~d}$, respectively. It is seen that, when $\lambda<1.1$, the response is localized around the left gap, although the localized modes involve motion of both gaps. This is due to the fact that nearly-simultaneous localized symmetric and antisymmetric modes give practically equal contribution to the response, so that motion disappears in the half of the string opposite to the driven end and disturbances cannot propagate. When $\lambda>1.1$, since the modes with close frequencies are extended, the disturbances propagate. 

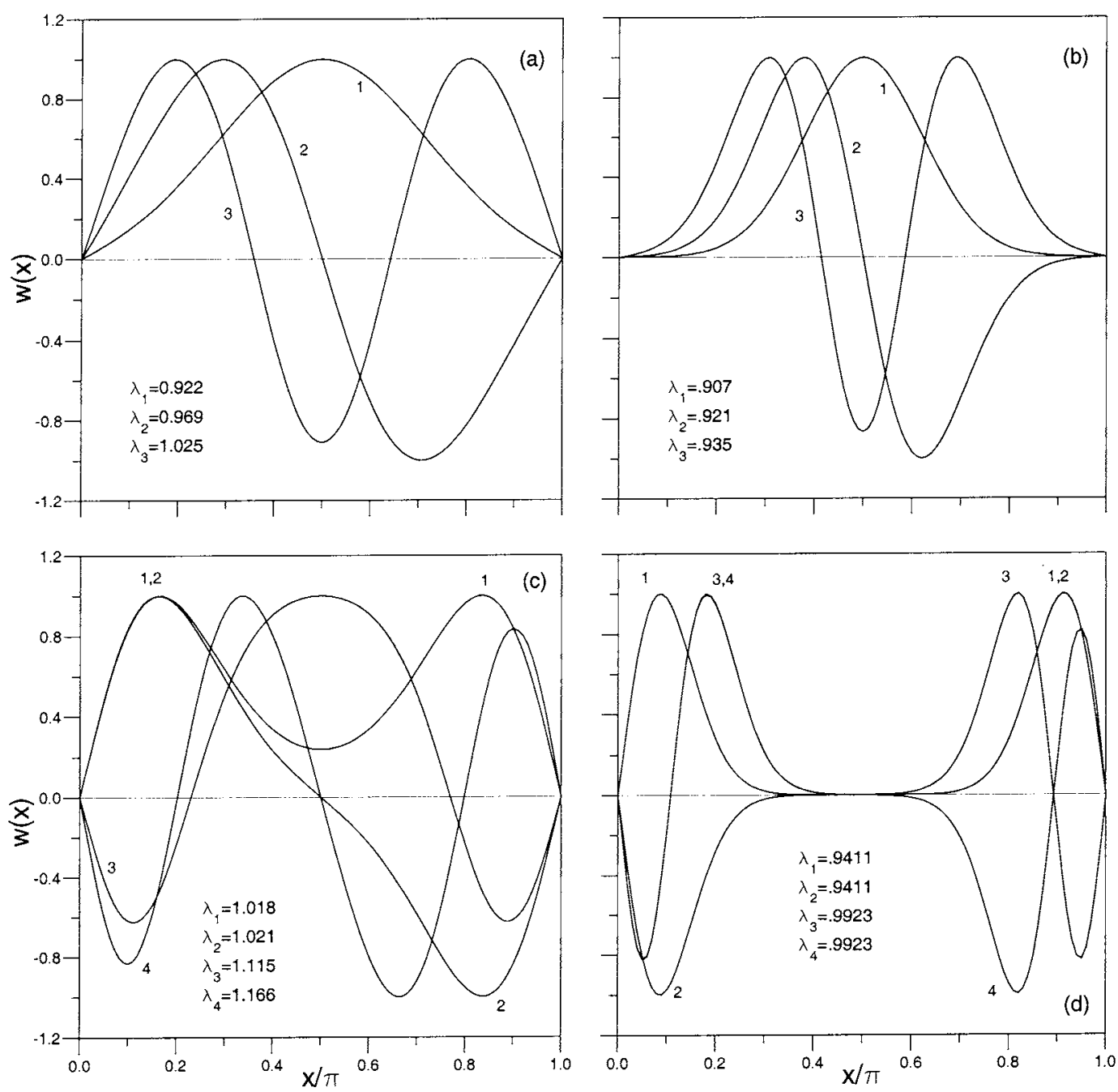

Figure 5. Normal modes for the string on elastic soil; (a, b) sinusoidal imperfections $f(x)=-\sin (x)$; (c, d) cosinusoidal imperfections $f(x)=-\cos (2 x) ; \varepsilon=0.1 ;(\mathrm{a}, \mathrm{c}) \beta=0.1,(\mathrm{~b}, \mathrm{~d}) \beta=10^{3 / 2}$.

\subsection{Free Vibrations of a Flexible Long Beam on Elastic Soil}

A problem similar to the foregoing is studied; here the string is replaced by a simply supported beam having small flexural stiffness $E I$. The relevant eigenvalue problem is

$$
\begin{aligned}
& E I w^{\prime \prime \prime \prime}+k_{f}(x) w-m_{0} \omega^{2} w=0, \\
& w(0)=w(l)=0, \quad w^{\prime \prime}(0)=w^{\prime \prime}(l)=0
\end{aligned}
$$

that, after having introduced the nondimensional quantities

$$
w=\frac{w}{l}, \quad \tilde{x}=\frac{\pi x}{l}, \quad \beta=\frac{\pi}{l} \sqrt[4]{\frac{E I}{k_{0}}}, \quad \lambda=\frac{m_{0} \omega^{2}}{k_{0}},
$$



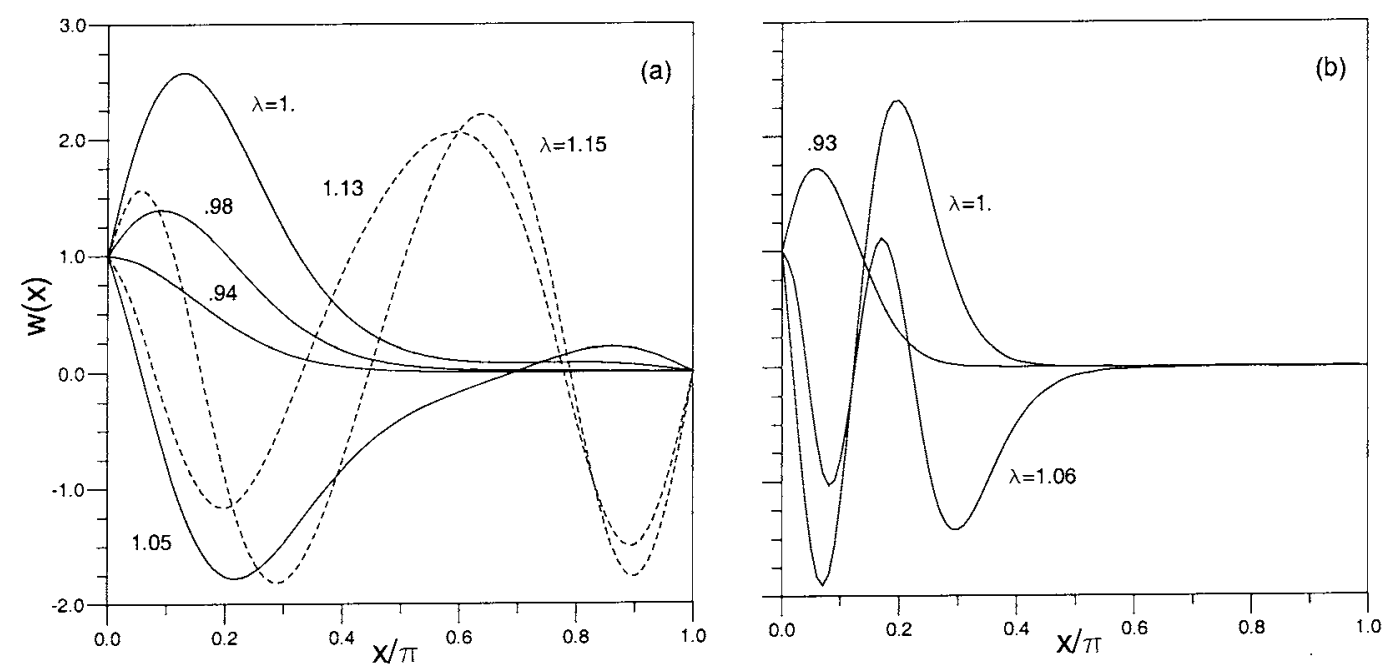

Figure 6. Time-harmonic response of the strings in Figures $5 \mathrm{c}$ and $5 \mathrm{~d}$ when the left support is driven with frequency $\lambda$.

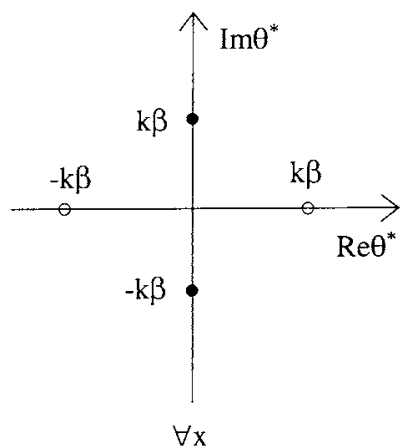

(a)

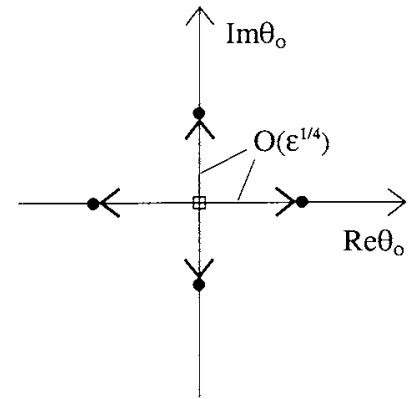

$\mathrm{f}(\mathrm{x})<\sigma$

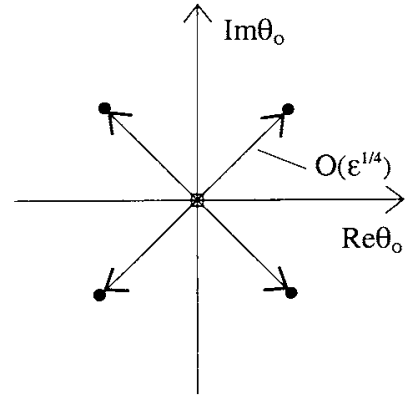

$f(x)>\sigma$

(b)

Figure 7. Characteristic exponents for the beam on elastic soil in the dynamic problem; (a) perfect system $(\varepsilon=0)$, (b) imperfect system $(\varepsilon \neq 0)$; $\square$ exponents at turning points.

reads

$$
\beta\left\{\begin{array}{l}
w_{1}^{\prime} \\
w_{2}^{\prime} \\
w_{3}^{\prime} \\
w_{4}^{\prime}
\end{array}\right\}=\left(\left\{\begin{array}{cccc}
0 & 1 & 0 & 0 \\
0 & 0 & 1 & 0 \\
0 & 0 & 0 & 1 \\
\lambda-1 & 0 & 0 & 0
\end{array}\right\}+\varepsilon\left(\begin{array}{cccc}
0 & 0 & 0 & 0 \\
0 & 0 & 0 & 0 \\
0 & 0 & 0 & 0 \\
-f(x) & 0 & 0 & 0
\end{array}\right)\right)\left\{\begin{array}{l}
w_{1} \\
w_{2} \\
w_{3} \\
w_{4}
\end{array}\right\}
$$

The characteristic exponents of the perfect system are $\theta_{1,2}^{*}= \pm i(\lambda-1)^{1 / 4}$ and $\theta_{3,4}^{*}=$ $\pm(\lambda-1)^{1 / 4}$. From the boundary conditions $\lambda_{k}^{*}=1+k^{4} \beta^{4}(k=1,2, \ldots)$ is found, i.e. $h=4$ in Equation (8); therefore $\theta_{1,2}^{*}= \pm i k \beta$ and $\theta_{3,4}^{*}= \pm k \beta$ (Figure 7a). The associated eigenfunctions are sinusoids, since the real exponents give no contribution; it results $\left|\theta_{k}^{*}\right|=O(\beta)$, as in the previous example. 
The imperfect system is now examined with $\varepsilon \geq O\left(\beta^{4}\right)$. The generating equation furnishes the following characteristic exponents

$$
\begin{aligned}
& \theta_{0_{1,2}}(x)= \pm i \varphi(x), \quad \theta_{0_{3,4}}(x)= \pm \varphi(x) \quad \text { if } f(x)<\sigma \\
& \theta_{0_{1,2}}(x)= \pm \frac{\sqrt{2}}{2}(1+i) \varphi(x), \quad \theta_{0_{3,4}}(x)= \pm \frac{\sqrt{2}}{2}(1-i) \varphi(x) \quad \text { if } f(x)>\sigma,
\end{aligned}
$$

with

$$
\varphi(x)=\sqrt[4]{\varepsilon|\sigma-f(x)|}
$$

and the right and left eigenvectors

$$
u_{0}=\left\{\begin{array}{llll}
1 & \theta_{0} & \theta_{0}^{2} & \theta_{0}^{3}
\end{array}\right\}^{T}, \quad v_{0}=\left\{\begin{array}{llll}
\bar{\theta}_{0}^{3} & \bar{\theta}_{0}^{2} & \bar{\theta}_{0} & 1
\end{array}\right\}^{T} .
$$

For different values of $x$, the characteristic exponents $\theta_{0}(x)$ are (a) two purely imaginary and two real or (b) two couples of complex conjugate numbers, with opposite real parts (Figure 7b). When $f(x)=\sigma$ the four eigenvalues coalesce at the origin and $x$ is a turning point. Since $0 \leq\left|\theta_{0}(x)\right| \leq O\left(\varepsilon^{1 / 4}\right)$, the imperfections modify the characteristic exponents of the perfect system by quantities of the same order as $\theta^{*}$ or larger; thus the eigenfunctions are strongly sensitive to the imperfections.

By following the procedure illustrated above the general solution

$$
\begin{aligned}
w(x)= & \frac{1}{[\sigma-f(x)]^{3 / 8}}\left[c_{1} \exp \psi_{1}(x)+c_{2} \exp \left(-\psi_{1}(x)\right)\right. \\
& \left.+c_{3} \sin \psi_{1}(x)+c_{4} \cos \psi_{1}(x)\right] \quad \text { if } f(x)<\sigma, \\
w(x)= & \frac{1}{[f(x)-\sigma]^{3 / 8}}\left\{\exp \psi_{2}(x)\left[c_{5} \sin \psi_{2}(x)+c_{6} \cos \psi_{2}(x)\right]\right. \\
& \left.+\exp \left(-\psi_{2}(x)\right)\left[c_{7} \sin \psi_{2}(x)+c_{8} \cos \psi_{2}(x)\right]\right\} \quad \text { if } f(x)>\sigma,
\end{aligned}
$$

is found, where

$$
\psi_{1}(x)=\int \sqrt[4]{\mu|f(x)-\sigma|} \mathrm{d} x, \quad \psi_{2}(x)=\frac{\sqrt{2}}{2} \psi_{1}(x)
$$

and $\mu=\varepsilon / \beta^{4} \geq O(1)$ have been posed. The solution is singular at the turning points.

The first three modes of vibration of a beam, numerically obtained by the Galerkin method, are shown in Figure 8 for the same imperfections considered in Figure 5 for the string. Although the values of $\mu$ in Figures $8 \mathrm{a}$ and $8 \mathrm{~b}$ are ten times larger than those in Figures $5 \mathrm{a}$ and $5 \mathrm{~b}$, the distortion of the modes is less important. This circumstance is explained by the fact that, by fixing $\varepsilon$ and $\mu$, i.e. by assuming that the values of $\beta^{2}$ and $\beta^{4}$ for the string and for the beam, respectively, are equal, the first system has higher modal density, due to the different powers of the number of mode $k$. The qualitative behavior is similar to that of the string, except in the neighborhood of the ends, where the modes are strongly exponentially modulated sinusoids. A zoom of the boundary region is illustrated in Figure 8c when $\mu$ is increased. When the cosinusoidal imperfection is considered for the lower $\mu$ (Figure $8 \mathrm{~d}$ ), the first two modes localize around the two gaps. The first mode is antisymmetric while the second is symmetric. 

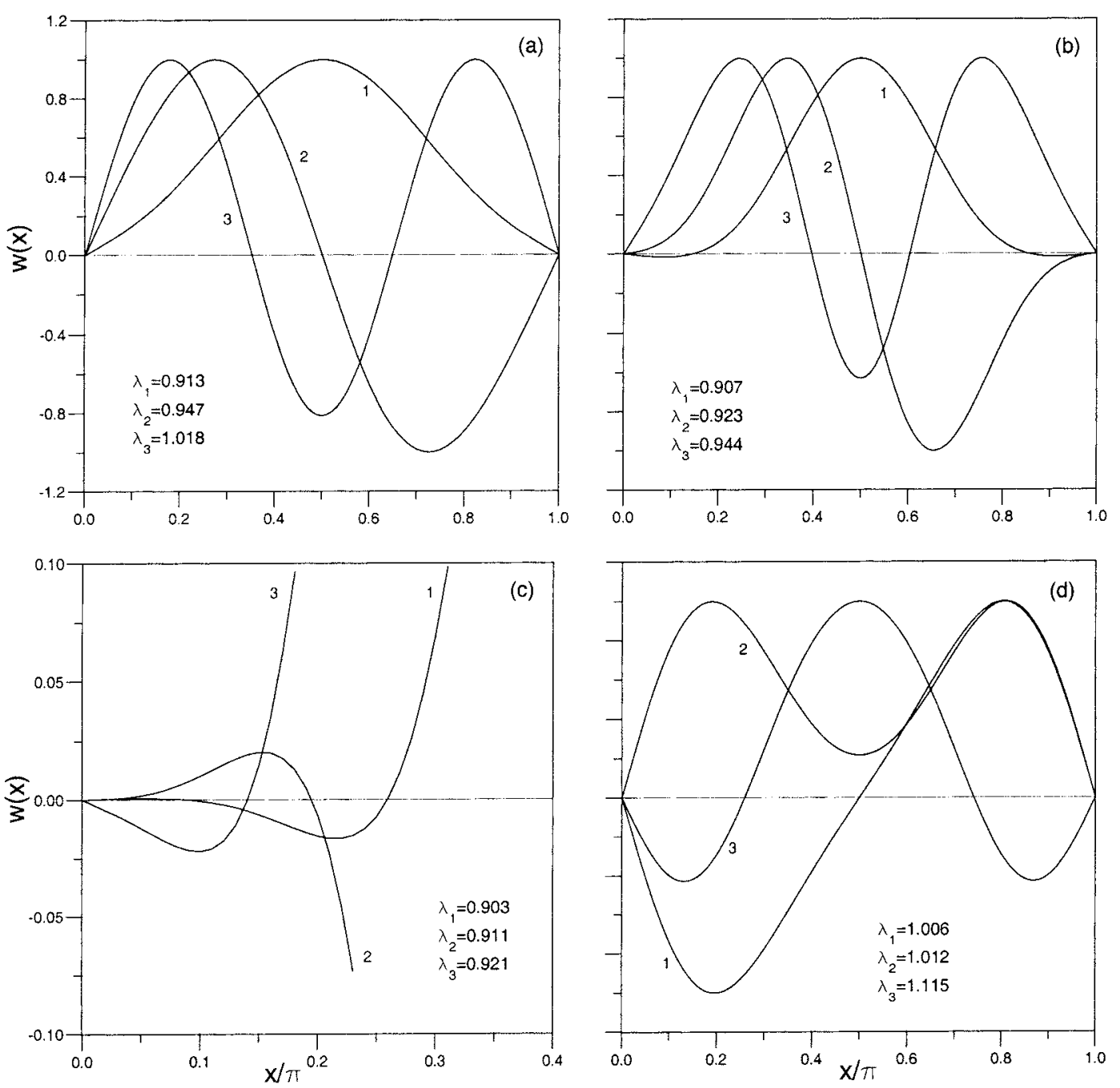

Figure 8. Normal modes of vibration for the beam on elastic soil; (a, b, c) sinusoidal imperfections $f(x)=-\sin (x)$, (d) cosinusoidal imperfections $f(x)=-\cos (2 x) ; \varepsilon=0.1 ;$ (a, d) $\beta=10^{-3 / 4}$, (b) $\beta=0.1$, (c) $\beta=10^{-5 / 4}$.

\subsection{Buckling of a Long Flexible Beam on Elastic Soil}

The buckling of a long beam with small flexural rigidity $E I$, simply supported at the ends, resting on Winkler soil, is considered. The beam is compressed by an axial force $\lambda N_{0}$ applied at one end and small longitudinal forces $\varepsilon p(x)$ distributed along the axis, having the character of defects. The soil, instead, is considered to be perfect, with constant stiffness $k_{0}$. The relevant eigenvalue problem reads

$$
\begin{aligned}
& E I w^{\prime \prime \prime \prime}+\left(N(x) w^{\prime}\right)^{\prime}+k_{0} w=0, \\
& w(0)=w(l)=0, \quad w^{\prime \prime}(0)=w^{\prime \prime}(l)=0 .
\end{aligned}
$$




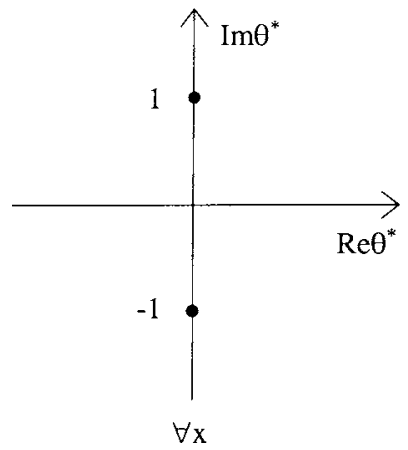

(a)
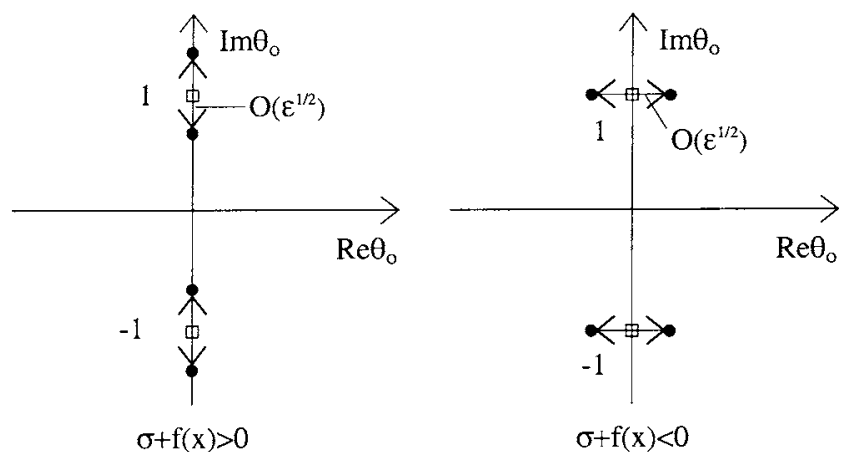

(b)

Figure 9. Characteristic exponents for the beam on elastic soil in the buckling problem; (a) perfect system $(\varepsilon=0)$, (b) imperfect system $(\varepsilon \neq 0)$; $\square$ exponents at turning points.

The axial stress at the abscissa $x$ can be expressed as

$$
N(x)=[\lambda+\varepsilon f(x)] N_{0},
$$

where $f(x)$ accounts for the shape of the imperfections. By assuming $N_{0}$ to be the critical load of the uniformly compressed beam, i.e. $N_{0}=2 \sqrt{k_{0} E I}$, and introducing the nondimensional quantities

$$
\tilde{w}=\frac{w}{l}, \quad \tilde{x}=\frac{\pi x}{l}, \quad \beta=\frac{\pi}{l} \sqrt[4]{\frac{E I}{k_{0}}} .
$$

Equation (31a) is rewritten as

$$
\beta\left\{\begin{array}{l}
w_{1}^{\prime} \\
w_{2}^{\prime} \\
w_{3}^{\prime} \\
w_{4}^{\prime}
\end{array}\right\}=\left(\left(\begin{array}{cccc}
0 & 1 & 0 & 0 \\
0 & 0 & 1 & 0 \\
0 & 0 & 0 & 1 \\
-1 & 0 & -2 \lambda & 0
\end{array}\right)+\varepsilon\left(\begin{array}{cccc}
0 & 0 & 0 & 0 \\
0 & 0 & 0 & 0 \\
0 & 0 & 0 & 0 \\
0 & 0 & -2 f(x) & 0
\end{array}\right)\right)\left\{\begin{array}{l}
w_{1} \\
w_{2} \\
w_{3} \\
w_{4}
\end{array}\right\},
$$

where the tilde has been omitted and $\beta \ll 1$ if $E I \ll k_{0} l^{4}$.

When the imperfections are absent the beam is uniformly compressed. The eigenvalues of the matrix $L^{*}$ are

$$
\theta_{1,2,3,4}^{*}= \pm i\left[\sqrt{\frac{\lambda+1}{2}} \pm \sqrt{\frac{\lambda-1}{2}}\right]
$$

When $\beta$ is small, it follows from the boundary conditions that $\lambda_{k}^{*} \cong 1+2 k^{2} \beta^{2}(k=$ $0,1, \ldots)$. Consequently, $\theta^{*}= \pm i(1 \pm k \beta)$ and the associated eigenfunctions are sinusoids with wavenumbers $n \pm k$, with $n \cong \beta^{-1} \gg 1$. The lower critical load $\lambda_{c}^{*}=1$ is associated with $k=$ 0 for which the characteristic exponents coalesce in twos (Figure 9a); the associated critical mode has $n$ halfwaves. The higher critical loads have value close to 1 and are associated with sinusoids having $n \pm k$ halfwaves, where $k \ll n$. The system has high modal density with exponent $h=2$; the eigenfunctions are fast varying, since $\left|\theta_{k}^{*}\right|=O(1)$. 
The imperfect system with $\varepsilon \geq O\left(\beta^{2}\right)$ is now considered. Equations (11a) admit the following eigenvalues

$$
\begin{array}{ll}
\theta_{0_{1,2,3,4}}(x)= \pm i\left[\sqrt{1+\frac{1}{2} \varepsilon|\sigma+f(x)|} \pm \sqrt{\frac{1}{2} \varepsilon|\sigma+f(x)|}\right] & \text { if } \sigma+f(x)>0, \\
\theta_{0_{1,2,3,4}}(x)= \pm\left[i \sqrt{1-\frac{1}{2} \varepsilon|\sigma+f(x)|} \pm \sqrt{\frac{1}{2} \varepsilon|\sigma+f(x)|}\right] \quad \text { if } \sigma+f(x)<0,
\end{array}
$$

associated with the right and left eigenvectors

$$
u_{0}=\left\{\begin{array}{llll}
1 & \theta_{0} & \theta_{0}^{2} & \theta_{0}^{3}
\end{array}\right\}^{T}, \quad v_{0}=\left\{\begin{array}{llll}
-\bar{\theta}_{0} & -1 & \bar{\theta}_{0}^{3} & \bar{\theta}_{0}^{2}
\end{array}\right\}^{T} .
$$

For different values of $x$ the characteristic exponents can be (a) purely imaginary or (b) two couples of complex conjugate, symmetric with respect the imaginary axis (Figure 9b). When $f(x)=-\sigma$ the four eigenvalues coalesce in twos and $x$ is a turning point. It should be noted that $1-O\left(\varepsilon^{1 / 2}\right) \leq\left|\theta_{0}(x)\right| \leq 1+O\left(\varepsilon^{1 / 2}\right)$, so that the imperfections produce only a small correction of order $\varepsilon^{1 / 2}$ of the characteristic exponents of the perfect system, unlike the systems studied before.

The solution can be remarkably simplified if terms of order $\varepsilon$ in Equations (36) are neglected in comparison with terms of order $\varepsilon^{1 / 2}$, i.e. if the following expressions for $\theta_{0}$ are used

$$
\begin{aligned}
& \theta_{0_{1,2,3,4}}(x)= \pm i\left[1 \pm \sqrt{\frac{1}{2} \varepsilon|\sigma+f(x)|}\right]+O(\varepsilon) \quad \text { if } \sigma+f(x)>0, \\
& \theta_{0_{1,2,3,4}}(x)= \pm\left[i \pm \sqrt{\frac{1}{2} \varepsilon|\sigma+f(x)|}\right]+O(\varepsilon) \quad \text { if } \sigma+f(x)<0 .
\end{aligned}
$$

After having determined $\theta_{1}(x)$ from Equation (12), the following solution for $w(x)$ is obtained

$$
\begin{aligned}
w(x)= & \frac{1}{\sqrt[4]{|\sigma+f(x)|}}\left\{\sin n x\left[c_{1} \sin \psi(x)+c_{2} \cos \psi(x)\right]\right. \\
& \left.+\cos n x\left[c_{3} \sin \psi(x)+c_{4} \cos \psi(x)\right]\right\} \quad \text { if } \sigma+f(x)>0, \\
w(x)= & \frac{1}{\sqrt[4]{|\sigma+f(x)|}}\left\{\sin n x\left[c_{5} \exp \psi(x)+c_{6} \exp (-\psi(x))\right]\right. \\
& \left.+\cos n x\left[c_{7} \exp \psi(x)+c_{8} \exp (-\psi(x))\right]\right\} \quad \text { if } \sigma+f(x)<0,
\end{aligned}
$$

where

$$
\psi(x)=\int \sqrt{\mu \frac{1}{2}|f(x)+\sigma|} \mathrm{d} x
$$

and $\mu=\varepsilon / \beta^{2} \geq O(1)$. It should be noted that Equation (39) furnishes sinusoidal functions with large wavenumber $n$, slowly modulated with the same law, sinusoidal or exponential, determined for the string (Equation (21)). The asymptotic solution is singular at the turning points. 

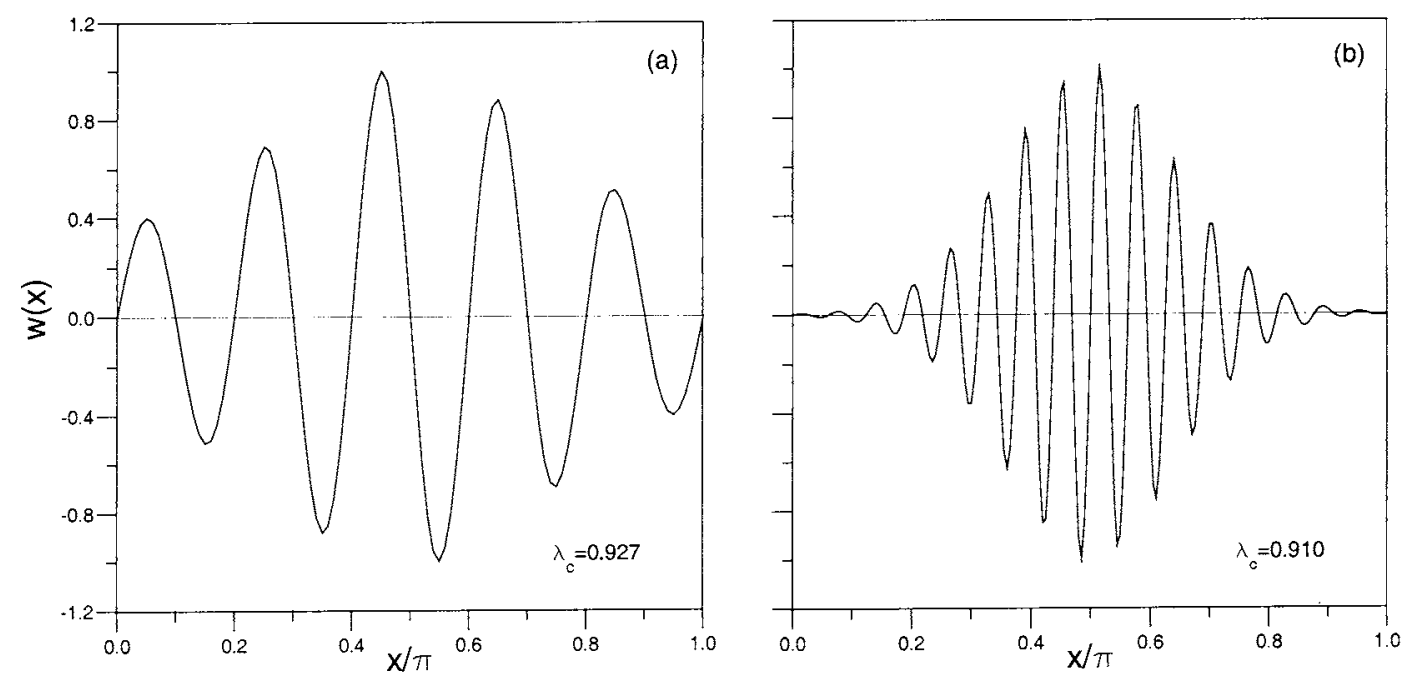

Figure 10. Critical buckling mode for the beam on elastic soil subjected to additional compression forces; $\varepsilon=0.1$, $f(x)=\sin (x)$; (a) $\beta=0.1$, (b) $\beta=10^{-3 / 2}$.

Figure 10 shows the buckling modes of two compressed beams, obtained numerically. Due to the increment of compression caused by the disturbance forces, the critical multiplier $\lambda_{c}$ is smaller than 1 ; its lower bound is $1-\varepsilon$, and it corresponds to a constant imperfection shape $f(x)=1$, i.e. to disturbance forces concentrated at the ends. Therefore, from Equation (18), $-1<\sigma<0$ and so $\sigma+f(x)$ is positive in the central region (sinusoidal modulation) and negative near the supports (exponential modulation). Thus, if $\mu$ is sufficiently large, the buckling mode localizes around the middle point of the beam, i.e. around the point of maximum stress.

\section{Remarks on the Characteristic Exponents Sensitivity}

It is useful to reconsider the generating equation (11a) and comment on the order of magnitude of the characteristic exponents $\theta^{*}$ and $\theta_{0}$ of perfect and imperfect systems, respectively.

By using Equation (5), the algebraic eigenvalue problem (11a) reads

$$
\left(L^{*}+\varepsilon L_{\varepsilon}-\theta_{0}\right) u_{0}=0 .
$$

This can be considered as an order- $\varepsilon$ perturbation of the eigenvalue problem (7), relative to the perfect system. Thus, it might be thought convenient to introduce the series expansions

$$
\theta_{0}=\theta^{*}+\varepsilon \theta_{\varepsilon}+O\left(\varepsilon^{2}\right), \quad u_{0}=u^{*}+\varepsilon u_{\varepsilon}+O\left(\varepsilon^{2}\right)
$$

and evaluate $\theta_{\varepsilon}, u_{\varepsilon}, \ldots$ through a standard perturbation method. However, this procedure would lead to incorrect results. In fact, in all the examples considered in the previous section, it has been observed that small imperfections of order $\varepsilon$ modify the eigenvalues $\theta^{*}$ of the system matrix $L^{*}$ by quantities of order greater than $\varepsilon$. In particular, the characteristic exponents in the dynamic problems have been modified by terms of order $\varepsilon^{1 / 2}$ and $\varepsilon^{1 / 4}$ (i.e. by corrections of the same order as the unmodified values $\theta^{*}$; see Figures 4 and 7) and in the buckling problem by terms of order $\varepsilon^{1 / 2}$ (while the unmodified values are of order 1; see Figure 9). 
In the perturbation theory of the eigenvalues it is known that these fractional powers appear when the unperturbed matrix is defective (i.e. does not have a complete set of eigenvectors) or nearly-defective [39-41]. Thus, defective matrices exhibit high sensitivity to imperfections.

It can be checked that the unperturbed matrices $L^{*}$ are in fact nearly-defective, because they have nearly-parallel eigenvectors. In particular, the matrices are themselves perturbations of order $\varepsilon$ of exactly defective matrices $L_{\mathrm{id}}$. In fact, if Equation (18) is substituted in the differential Equations (16), (25) or (34) and the term $\varepsilon \sigma$ is properly shifted in the perturbation matrix $\varepsilon L_{\varepsilon}$, the resulting order-one matrix $L_{\text {id }}$ is found to be defective. In the first two problems, $L_{\mathrm{id}}$ is a Jordan block of order 2 or 4 , admitting therefore a unique eigenvector associated with the multiple zero eigenvalue; in the buckling problem, $L_{\text {id }}$ admits two double eigenvalues $\theta_{\mathrm{id}}= \pm i$, each associated with a unique eigenvector.

It has been shown by the author in [42] that the most appropriate method to study perturbations of nearly-defective matrices $L^{*}$ is to build an exactly defective ideal matrix $L_{\mathrm{id}}$, close in some sense to the original matrix $L^{*}$, and then to perturb directly $L_{\mathrm{id}}$, by applying the algorithm developed in [41]. Here the ideal matrix $L_{\text {id }}$ appears in a natural way in the problem. An example of application of the method is given in the Appendix.

These considerations permit the localization phenomenon to be considered in a different perspective. Namely, since nearly defective continuous structures are strongly sensitive to imperfections, small perturbations cause large variations of the characteristic exponents; in particular, imaginary exponents can become complex and consequently the associated periodic modes localize.

\section{Conclusions}

Localization phenomena in one-dimensional imperfect continuous structures have been analyzed, both in dynamics and buckling. A general perturbation method has been developed which generalizes the classical WKB method. The method has been applied to analyze the dynamics of a string and the dynamics and buckling of a beam on elastic soil. All these structures have been found to have nearly-defective system matrices, so their characteristic exponents are highly sensitive to imperfections.

The following conclusions are drawn.

1. In engineering structures, the most important mechanism of localization calls for the occurrence of turning points at which the eigenfunctions change from oscillating to exponential or vice versa.

2. Localization in continuous systems of finite length occurs when the ratio between the amplitude of the imperfections and the coupling comes to have sufficiently large values. Only the first modes localize and their number increases when the imperfection/coupling ratio increases.

3. In the dynamic problem, harmonic disturbances cannot propagate through the structure if their frequency is close to a localized mode natural frequency; similarly, in the buckling problem, disturbances cannot propagate if the axial load is close to a critical load associated with a localized buckling pattern.

4. Interaction among nearly-simultaneous buckling modes of perfect structures in the nonlinear field can lead to localized buckling patterns. The phenomenon reveals close analogies with the localization of linear imperfect systems. 
5. Asymptotic solutions can be built up by applying the perturbation method illustrated; this transforms the differential problem into a sequence of algebraic problems in which the spatial variable appears as a parameter.

6. Localization of vibrations of a string (or beam) on elastic soil is due to characteristic exponents changing from purely imaginary to real (from purely imaginary to complex conjugates, with the real and imaginary parts equal in modulus); localization of the buckling mode of a beam on elastic soil is due to characteristic exponents changing from purely imaginary to complex conjugates with small real part.

7. Systems with nearly defective matrices are highly sensitive; in fact, small perturbations of order $\varepsilon$ produce modifications of the characteristic exponents of order $\varepsilon^{1 / 2}$ or larger. This effect exalts localization.

\section{Appendix: An Example of Solution of a Nearly Defective Eigenvalue Problem}

As an example of application of the method outlined in Section 5, the eigenvalue problem (41), relative to the buckling problem dealt with in Section 4.3, is solved. The ideal defective matrix $L_{\mathrm{id}}$ is introduced and the perturbation matrix $L_{\varepsilon}$ is redefined as follows

$$
L_{\mathrm{id}}=\left(\begin{array}{cccc}
0 & 1 & 0 & 0 \\
0 & 0 & 1 & 0 \\
0 & 0 & 0 & 1 \\
-1 & 0 & -2 & 0
\end{array}\right), \quad L_{\varepsilon}=\left(\begin{array}{cccc}
0 & 0 & 0 & 0 \\
0 & 0 & 0 & 0 \\
0 & 0 & 0 & 0 \\
0 & 0 & -2(\sigma+f) & 0
\end{array}\right)
$$

in such a way that $L=L_{\mathrm{id}}+\varepsilon L_{\varepsilon}$ (see Equation (36)). By introducing the series expansions [41]

$$
\begin{aligned}
& \theta_{0}=\theta_{\mathrm{id}}+\varepsilon^{1 / 2} \theta_{\varepsilon}+\varepsilon \theta_{\varepsilon \varepsilon}+O\left(\varepsilon^{3 / 2}\right), \\
& u_{0}=i_{\mathrm{id}}+\varepsilon^{1 / 2} u_{\varepsilon}+\varepsilon u_{\varepsilon \varepsilon}+O\left(\varepsilon^{3 / 2}\right),
\end{aligned}
$$

the following perturbation equations are obtained

$$
\begin{aligned}
& \left(L_{\mathrm{id}}-\theta_{\mathrm{id}}\right) u_{\mathrm{id}}=0, \\
& \left(L_{\mathrm{id}}-\theta_{\mathrm{id}}\right) u_{\varepsilon}=\theta_{\varepsilon} u_{\mathrm{id}}, \\
& \left(L_{\mathrm{id}}-\theta_{\mathrm{id}}\right) u_{\varepsilon \varepsilon}=-L_{\varepsilon} u_{\mathrm{id}}+\theta_{\varepsilon} u_{\varepsilon}+\theta_{\varepsilon \varepsilon} u_{\mathrm{id}} .
\end{aligned}
$$

The right and left eigenvectors of $L_{\mathrm{id}}$ associated with $\theta_{\mathrm{id}}= \pm i$ are

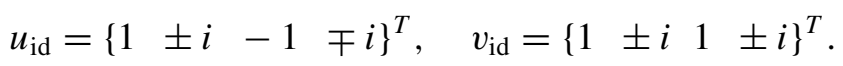

The perturbation equation (45b) admits the following particular solution

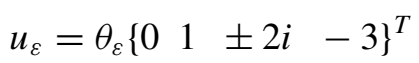

in which $\theta_{\varepsilon}$ is undetermined. By imposing the solvability condition to Equation (45c), two solutions for $\theta_{\varepsilon}$ are obtained

$$
\theta_{\varepsilon}= \pm \sqrt{-\frac{1}{2}(f+\sigma)} .
$$


The series expansions (44) coincide with the exact solution determined in Section 4.3 and then expanded up to $\varepsilon^{1 / 2}$-order terms.

\section{References}

1. Matsuda, H. and Ishii, K., 'Localization of normal modes and energy transport in the disordered harmonic chain', Supplement of the Progress of Theoretical Physics 45, 1970, 56-86.

2. Ishii, K., 'Localization of eigenstates and transport phenomena in the one-dimensional disordered system', Supplement of the Progress of Theoretical Physics 53, 1973, 77-138.

3. Hodges, C. H., 'Confinement of vibration by structural irregularity', Journal of Sound and Vibration 82, 1982, 411-424.

4. Li, D. and Benaroya, H., 'Dynamics of periodic and near-periodic structures', Applied Mechanics Review 45(11), 1982, 447-459.

5. Benaroya, H., 'Localization and effects of irregularities in structures', Applied Mechanics Review 49, 1996, 57-135.

6. Pierre, C. and Dowell, E. H., 'Localization of vibrations by structural irregularity', Journal of Sound and Vibration 114(3), 1987, 549-564.

7. Bendiksen, O., 'Mode localization phenomena in large space structures', AIAA Journal 25(9), 1987, 12411248.

8. Pierre, C., 'Mode localization and eigenvalue loci veering phenomena in disordered structures', Journal of Sound and Vibration 126(3), 1988, 485-502.

9. Pierre, C., 'Weak and strong vibration localization in disordered structures: A statistical investigation', Journal of Sound and Vibration 139(1), 1990, 111-132.

10. Wu, G., 'Free vibration of cyclic assemblies with a single disordered component', Journal of Sound and Vibration 165(3), 1993, 567-570.

11. Ottarsson, G. and Pierre, C., 'A transfer matrix approach to free vibration localization in mistuned blade assemblies', Journal of Sound and Vibration 197(5), 1996, 589-618.

12. Hodges, C. H. and Woodhouse, J., 'Vibration isolation from irregularity in a nearly periodic structure: Theory and measurements', Journal of Acoustical Society of America 79(9), 1983, 894-905.

13. Cornwell, P. J. and Bendiksen, O., 'Localization of vibrations in large space reflectors', AIAA Journal 27(2), 1989, 219-226.

14. Pierre, C. and Cha, P. D., 'Strong mode localization in nearly periodic disordered structures', AIAA Journal 27(2), 1989, 227-241.

15. Al-Jawi, A. A., Ulsoy, A. G., and Pierre, C., 'Vibration localization in dual-span, axially moving beams', Journal of Sound and Vibration 179(2), 1995, 243-266 (part I), 267-287 (part II).

16. Keane, A. J. and Price, W. G., 'On the vibrations of mono-coupled periodic and near-periodic structures', Journal of Sound and Vibration 128(3), 1989, 423-450.

17. Cai, G. Q. and Lin, Y. K., 'Localization of wave propagation in disordered periodic structures', AIAA Journal 29, 1990, 450-456.

18. Lin, Y. K. and Cai, G. Q., 'Disordered periodic structures', in Computational Stochastic Mechanics, C. A. Brebbia and P. Spanos (eds.), Elsevier, Amsterdam, 1991, pp. 777-788.

19. Cha, P. D. and Pierre, C., 'Vibration localization by disorder in assemblies of monocoupled multimode component systems', Journal of Applied Mechanics 58, 1991, 1072-1081.

20. Cheng, S. P. and Perkins, N. C., 'Closed-form vibration analysis of sagged cables/mass suspension', ASME Paper 92WA/APM-7, 1992.

21. Bouzit, D. and Pierre, C., 'Localization of vibration in disordered multi-span beams with damping', Journal of Sound and Vibration 187(4), 1995, 625-648.

22. Scott, J. M. F., 'The statistics of waves propagating in a one-dimensional random medium', Proceedings of Royal Society of London A398, 1985, 341-363.

23. Luongo, A., 'Mode localization by structural imperfections in one-dimensional continuous systems', Journal of Sound and Vibration 155(2), 1992, 249-271.

24. Tjavaras, A. A. and Triantafyllou, M. S., 'Non-linear response of two disordered pendula', Journal of Sound and Vibration 190(1), 1996, 65-76. 
25. Zevin, A. A., 'Localization of periodic oscillations in discrete non-linear systems', Journal of Sound and Vibration 193(4), 1996, 847-862.

26. Vakakis, A., Nayfeh, T., and King, M., 'A multiple-scales analysis of nonlinear, localized modes in a cyclic periodic system', Transactions of the ASME 60, 1993, 388-397.

27. Emaci, E., Nayfeh, T. A. and Vakakis, A. F. ,'Numerical and experimental study of nonlinear localization in a flexible structure with vibro-impacts', Journal of Applied Mathematics and Mechanics (ZAMM) 77(7), 1997, 527-541.

28. Nayfeh, T. A., Emaci, E. and Vakakis, A. F., 'Application of nonlinear localization to the optimization of a vibration isolation system', AIAA Journal 35(8), 1997, 1378-1386.

29. Pierre, C., 'Curve veering and mode localization in a buckling problem', Journal of Applied Mathematics and Physics (ZAMP) 40, 1989, 758-761.

30. Gioncu, V., 'General theory of coupled instabilities', Thin Walled Structures 19(2-4), 1994, 81-127.

31. Luongo, A., 'On the amplitude modulation and localization phenomena in interactive buckling problems', International Journal of Solids and Structures 27(15), 1991, 1943-1954.

32. Nayfeh, A. H., Perturbation Methods, Wiley, New York, 1973.

33. Tvegaard, V. and Needleman, A., 'On the localization of buckling patterns', Journal of Applied Mechanics 47, 1980, 613-619.

34. Tvegaard, V. and Needleman, A., 'On the development of localized buckling patterns', in Collapse, The Buckling of Structures in Theory and Practice, J. M. T. Thompson and G. W. Hunt (eds.), Cambridge University Press, Cambridge, 1983, pp. 1-17.

35. Potier-Ferry, M., 'Amplitude modulation, phase modulation and localization of buckling patterns', in Collapse, The Buckling of Structures in Theory and Practice, J. M. T. Thompson and G. W. Hunt (eds.), Cambridge University Press, Cambridge, 1983, pp. 149-159.

36. Luongo, A. and Pignataro, M. ,'Multiple interaction and localization phenomena in the postbuckling of compressed thin-walled members', AIAA Journal 26(11), 1988, 1395-1402.

37. Hunt, G. W., Bolt, H. M., and Thompson, J. M. T., 'Structural localization phenomena and the dynamical phase-space analogy', Proceedings of Royal Society London A425, 1989, 245-267.

38. Luongo, A., 'Perturbation methods for the eigenvalue analysis of imperfect systems with high modal density', Parts I and II, Accademia Peloritana dei Pericolanti, Messina (Italy), Vol. LXVIII, 401-423, 1991, 424-447 [in Italian].

39. Wilkinson, J. H., The Algebraic Eigenvalue Problem, Oxford University Press, London, 1965.

40. Kato, T., A Short Introduction to Perturbation Theory for Linear Operators, Springer-Verlag, New York, 1982.

41. Luongo, A., 'Eigensolutions sensitivity for nonsymmetric matrices with repeated eigenvalues', AIAA Journal 31(7), 1994, 1321-1328.

42. Luongo, A., 'Eigensolutions of perturbed nearly-defective matrices', Journal of Sound and Vibration 185(3), 1995, 377-395. 